\title{
Diagnosis of Anomalous Origin of the Right Subclavian Artery from the Right Pulmonary Artery in a Patient with D-transposition of the Great Arteries Utilizing Transthoracic Echocardiography
}

\author{
Luv Makadia ${ }^{1}$, James Nielsen ${ }^{1}$, Susheel Kumar ${ }^{2}$, and Puneet Bhatla ${ }^{3}$ \\ ${ }^{1}$ Hassenfeld Children's Hospital at NYU Langone \\ ${ }^{2}$ NYU Langone Health \\ ${ }^{3} \mathrm{NYU}$ Langone Medical Center
}

June 15, 2020

\begin{abstract}
Diagnosis of anomalous origin of the right subclavian artery (AORSA) from the right pulmonary artery (RPA) is usually made using CT or invasive angiography. We report a patient diagnosed using transthoracic echocardiography (TTE). A newborn girl prenatally known to have d-TGA presented with cyanosis sparing the right hemithorax and arm. Oxygen saturations on the right hand were persistently higher than on the right ear and other extremities. Repeat TTE using a modified echocardiographic imaging plane allowed for full visualization of the entire subclavian artery course, revealing AORSA from RPA. We discuss further the approach to echocardiographic diagnosis and surgical implications.
\end{abstract}

\section{Introduction}

Anomalous origin of the right subclavian artery (AORSA) from the right pulmonary artery (RPA) is extremely rare in dextro-transposition of the great arteries (d-TGA). Only a few cases have been reported. ${ }^{1-6}$ Diagnosis is usually incidentally made using Computed Tomography (CT) or invasive angiography. We report a patient that was interestingly suspected based on clinical presentation and subsequently diagnosed with transthoracic echocardiography (TTE). This case demonstrates the importance of considering aortic arch anomalies in d-TGA when patients do not show reverse differential cyanosis. We also discuss the clinical significance, embryology, approach to echocardiographic diagnosis, and surgical implications.

\section{Case Report}

We present the case of a 2,770 gram baby girl born at 37 weeks and 2 days with a prenatal diagnosis of d-TGA with intact ventricular septum who was initially brought to the neonatal intensive care unit on prostaglandin. She was then quickly taken for a cardiac catheterization for a balloon atrial septostomy. TTE confirmed the prenatal diagnosis of d-TGA with intact ventricular septum and also showed a large left patent ductus arteriosus (PDA) with bidirectional shunting. Aortic arch imaging suspected AORSA because of a lack of bifurcation of the first right brachiocephalic branch, although its origin was not visualized. Her body appeared cyanotic and dusky with sparing of the right hemithorax and arm. Pre-ductal oxygen saturation on the right hand was consistently above $90 \%$ whereas the post-ductal oxygen saturations on all other extremities were $60-70 \%$. Interestingly, when the pre-ductal pulse oximeter was moved to the right ear, the oxygen saturation dropped to resemble the post-ductal levels. This prompted for systematic evaluation for the origin of the right subclavian artery from the pulmonary artery system, in the setting of transposition physiology. Repeat TTE confirmed prior findings and clearly demonstrated the right subclavian artery from the proximal RPA. Additional anatomic details included anomalous origin of the left coronary 
artery from the right sinus and an isolated cleft of the mitral valve that was diagnosed on fetal imaging. On day of life 13, the patient underwent an arterial switch operation (ASO) with LeCompte maneuver, single coronary button with creation of a neo-aortic to coronary hood, closure of atrial septal defect, and ligation of the left-sided PDA. The preoperative echocardiographic diagnosis of AORSA from the proximal RPA was confirmed (Figure 1). The anomalous vessel was ligated and divided from the RPA. She had an uncomplicated post-operative course.

\section{Discussion}

In d-TGA, failed spiraling during great artery septation causes the pulmonary and systemic circulations to exist in parallel. Newborns with physiologically elevated pulmonary vascular resistance have pulmonary to systemic flow through the PDA resulting in reverse differential cyanosis. Our patient does not show reverse differential cyanosis. Instead, she demonstrates persistently elevated right arm saturations as compared to the right ear and other extremities. This, along with cyanosis that very typically spared the right arm and hemithorax, strongly raised the suspicion for an anomaly with the aortic arch branching, specifically that the right subclavian could be originating from the pulmonary circuit and carrying oxygenated blood.

AORSA from the RPA can also be understood embryologically. In normal embryonic development, the right $7^{\text {th }}$ intersegmental artery travels cranially and becomes the right subclavian artery, eventually coming off of the brachiocephalic artery which itself arises from the $4^{\text {th }}$ aortic arch. ${ }^{7,8}$ The extrapericardial portion of the pulmonary arteries arises from the $6^{\text {th }}$ aortic arch. ${ }^{9}$ Regression of the distal right $4^{\text {th }}$ and $6^{\text {th }}$ arches allows the left $4^{\text {th }}$ arch to become the aortic arch and the left $6^{\text {th }}$ arch to become the PDA. When the right $6^{\text {th }}$ arch persists, it forms the right ductus arteriosus and pulmonary artery, potentially leading to an AORSA from the RPA. ${ }^{3}$

Our report emphasizes the difficulty in visualizing AORSA from the RPA, something that was missed on the initial TTE and also which most other reports have described to be diagnosed using a CT or invasive angiogram. TTE interrogation has classically depended on the suprasternal axial plane sweeping superiorly to define the aortic arch sidedness and branching. This traditional imaging plane, though adequate in raising the suspicion of AORSA, fails to itself evaluate for AORSA from the PA. We recommend a modified imaging plane to evaluate for this lesion, where the suprasternal axial arch view is started with the transducer aiming more inferiorly such that the distal main and proximal right PAs are visualized. Careful slow sweep of the transducer to an axial view and then oblique coronal view should be performed with color comparison (Figure 2). This imaging plane can clearly demonstrate the origin, proximal and distal course of the AORSA, including its relationship to the aorta and presence or absence of the right sided PDA (Figure 3). Once this is obtained, an orthogonal sagittal plane can be obtained to visualize the AORSA in a long axis (Figure 4).

Effective diagnosis of AORSA from the RPA is crucial because, if missed, may result in steal of blood away from the subclavian artery and to the pulmonary system. Some reports have described surgical correction with ASO with subclavian artery ligation and division from the RPA (our patient), ${ }^{2,4}$ but this carries the risk of subclavian steal syndrome where the subclavian artery is supplied by the vertebral artery. Alternatively, reports have described ASO with subclavian artery re-implantation to the systemic circulation, usually the aorta or common carotid. ${ }^{1,3}$ Although this is more physiologic, the mobilization of the subclavian artery is technically challenging and there is an increased risk of restenosis.

\section{Conclusions}

D-TGA patients who do not have reverse differential cyanosis must be investigated for aortic arch anomalies, particularly AORSA from the pulmonary circulation. Because the full course of an AORSA is difficult to visualize using TTE, the diagnosis is traditionally made by CT or invasive angiography. We present and recommend a modified echocardiographic imaging plane to visualize both the proximal and distal course of an AORSA, ultimately allowing for safe and non-invasive diagnosis.

\section{Author Contributions}


All authors helped conceptualize the study. LDM drafted the article, which all authors helped revise and finalize.

Acknowledgments: The authors thank Dr. Susheel Kumar for sketching Figure 1.

\section{References}

1. Talwar S, Gupta A, Choudhary SK: d-transposition of great arteries, dextrocardia with aberrant origin of right subclavian artery from pulmonary artery. J Card Surg 2018:33:691-692.

2. Park H, Song J, Huh J, et al: Anomalous Origin of the Right Subclavian Artery in a Patient with D-transposition of the Great Arteries. Korean J Thorac Cardiovasc Surg 2018:51:403-405.

3. Arunamata A, Perry SB, Kipps AK, et al: Isolation of the right subclavian artery in a patient with d-transposition of the great arteries. Ann Pediatr Cardiol 2015:8:161-3.

4. Mosieri J, Chintala K, Delius RE, et al: Abnormal origin of the right subclavian artery from the right pulmonary artery in a patient with D-transposition of the great vessels and left juxtaposition of the right atrial appendage: An unusual anatomical variant. J Card Surg 2004:19:41-4.

5. Paquet M, Williams RL: Origin of the right subclavian artery from the right pulmonary artery in a newborn with complete transposition of the great arteries. Can J Cardiol 1994:10:932-4.

6. Marin C, Sanchez ML, Fernandez-Velilla M, et al: MR imaging of isolated right subclavian artery. Pediatr Radiol 2008:38:216-9.

7. Hernandez LE, Shepard CW, Bamforth SD, et al: The right subclavian artery arising as the first branch of a left-sided aortic arch. World J Pediatr Congenit Heart Surg 2014:5:456-9. 8.

8. Gupta SK, Bamforth SD, Anderson RH: How frequent is the fifth arch artery? Cardiol Young 2014:29:1-19.

9. Anderson RH, Chaudhry B, Mohun TJ, et al: Normal and abnormal development of the intrapericardial arterial trunks in humans and mice. Cardiovasc Res 2012:95:108-15.

\section{Figure Legends}

Figure 1. 1) d-TGA: aorta is seen arising anterior to the pulmonary artery with a left aortic arch; first branch of the aortic arch is the right common carotid artery; second branch is the left common carotid artery; third branch is the left subclavian artery,2) AORSA: right subclavian artery arises from the proximal RPA and has a superior and leftward course with close proximity to the distal ascending aorta before finally coursing rightward, 3) large left sided PDA.

$($ Ao $=$ aorta, AORSA $=$ anomalous origin of the right subclavian artery, d-TGA $=$ d-transposition of the great arteries, LPA = left pulmonary artery, $\mathrm{PA}=$ pulmonary artery, $\mathrm{PDA}=$ patent ductus arteriosus, $\mathrm{RPA}$ = right pulmonary artery)

Figure 2. The following supplementary material is available online - Movie Clip. Transthoracic echocardiogram with modified imaging plane with color comparison demonstrating the origin and course of the AORSA.

$($ AORSA $=$ anomalous origin of the right subclavian artery)

Codec used: mpeg4

Figure 3. Two-dimensional (2D) TTE suprasternal notch oblique axial image with color comparison. This image is obtained by an inferiorly directed angulation of the transducer from a standard straight axial plane such that the ascending aorta is seen in a cross-section and the LPA and PDA are seen in a long axis (oblique coronal plane). This enables us to visualize the distal MPA and the proximal RPA, which is most frequently the region of the AORSA from the PA. AORSA is visualized just rightward to the PDA connection at and around the junction of RPA and MPA (red arrowhead). 
$($ AORSA $=$ anomalous origin of the right subclavian artery, LPA $=$ left pulmonary artery, MPA $=$ main pulmonary artery, $\mathrm{PA}=$ pulmonary artery, $\mathrm{PDA}=$ patent ductus arteriosus, $\mathrm{RPA}=$ right pulmonary artery)

Figure 4. Two-dimensional (2D) TTE high right parasternal oblique sagittal image. This image is obtained by rotating the transducer perpendicular to the initial axial plane. This enables us to visualize the distal MPA, LPA and the origin of AORSA in a long axis plane and just rightward of the ascending aorta. Ascending aorta is seen superiorly and separate from the RSCA in a long axis plane.

$($ AORSA $=$ anomalous origin of the right subclavian artery, LPA $=$ left pulmonary artery, MPA $=$ main pulmonary artery, RSCA = right subclavian artery)

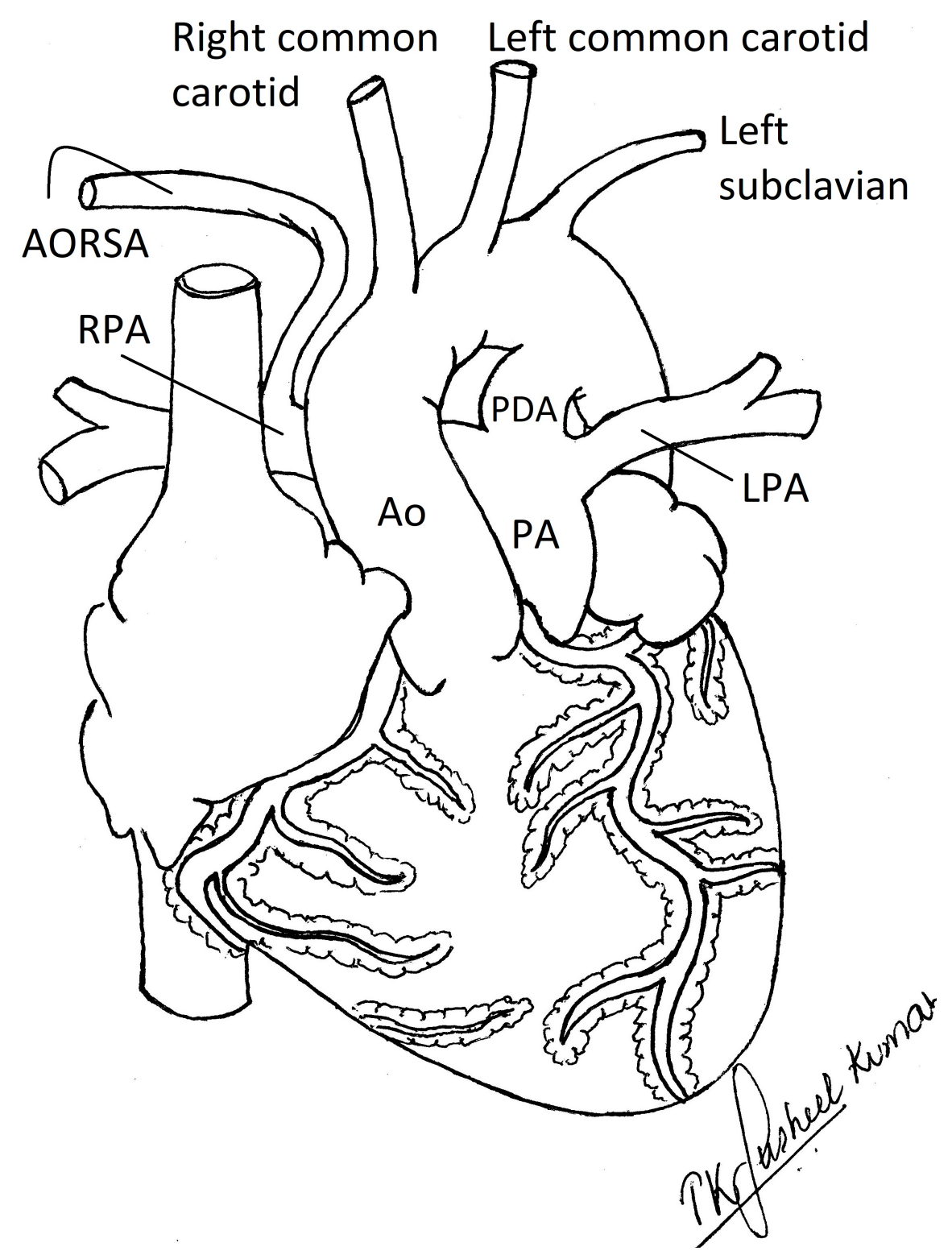



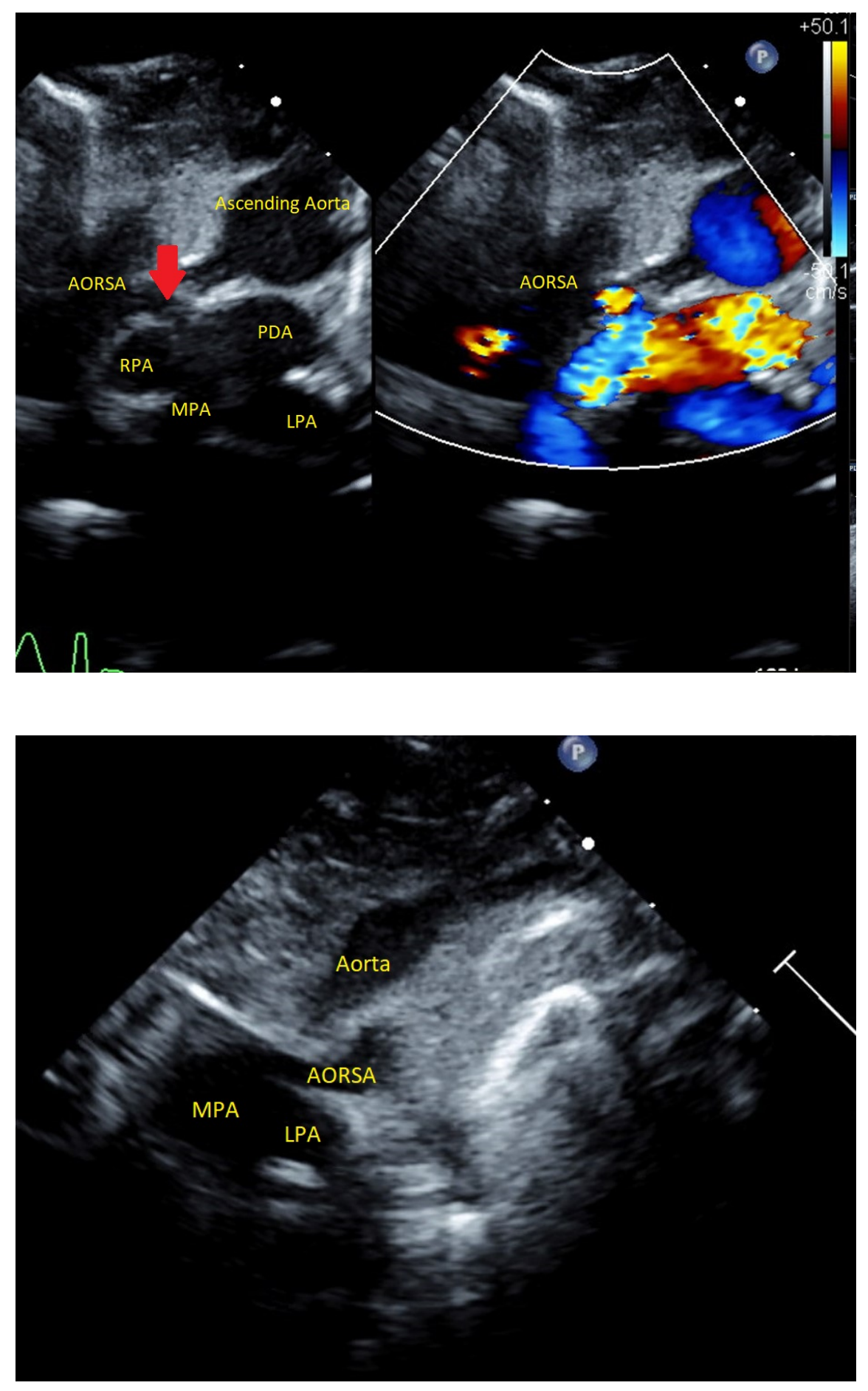